\section{Vol. 38, Issue 6, December 2011}

Editorial

349 Tissue Banking and Transfusion Medicine a Suitable Cooperation Model

Pruß, A. (Berlin)

\section{Original Articles}

352 The EUROCET Network: Support for Coding, Vigilance and Surveillance

Mareri, M.; Filippetti, M.; Ghirardini, A.; Vespasiano, F.; Di Ciaccio, P.; Nanni Costa, A. (Rome)

357 A Q Fever Outbreak in the Netherlands: Consequences for Tissue Banking

Van Wijk, M.J. (Leiden); Hogema, B.M. (Amsterdam);

Maas, D.W.; Bokhorst, A.G. (Leiden)

365 Validation of the Serological Testing for Anti-HIV-1/2, Anti-HCV, HBsAg, and Anti-HBc from Postmortem Blood on the Siemens-BEP-III Automatic System

Kalus, U.; Wilkemeyer, I,; Caspari, G.; Schroeter, J.; Pruss, A. (Berlin)

373 An Analysis of Bone Donor Deferral Rates in Scotland - a 6-Year Study

Galea, G. (Edinburgh)

379 Determination of Residual Antibiotics in Cryopreserved Heart Valve Allografts

Jashari, R.; Faucon, F.; Van Hoeck, B.; De Gelas, S.; Fan, Y.;

Vandenbulcke, S. (Brussels)

387 Impact of Hypothermia upon Chondrocyte Viability and Cartilage Matrix Permeability after 1 Month of Refrigerated Storage

Brockbank, K.G.M. (North Charleston, SC/Atlanta, GA Charleston, SC); Rahn, E. (North Charleston, SC); Wright, G.J. (Charleston, SC); Chen, Z. (North Charleston, SC); Yao, H. (Charleston, SC)

\section{Band 38, Heft 6, Dezember 2011}

Editorial

349 Gewebebanken und Transfusionsmedizin ein geeignetes Kooperationsmodell Pruß, A. (Berlin)

Originalarbeiten

352 Das EUROCET-Netzwerk: Unterstützung für Kodierung, Vigilanz und Überwachung

Mareri, M.; Filippetti, M.; Ghirardini, A.; Vespasiano, F.; Di Ciaccio, P.; Nanni Costa, A. (Rome)

357 Ein Q-Fieberausbruch in den Niederlanden: Konsequenzen für die Gewebebanken Van Wijk, M.J. (Leiden); Hogema, B.M. (Amsterdam); Maas, D.W.; Bokhorst, A.G. (Leiden)

365 Validierung der serologischen Testung für AntiHIV-1/2, Anti-HCV, HBsAg und Anti-HBc von postmortalem Blut mit Hilfe des Siemens-BEP-III Automatic System

Kalus, U.; Wilkemeyer, I,; Caspari, G.; Schroeter, J.; Pruss, A. (Berlin)

373 Eine Analyse von Knochenspender-

Rückweisungsraten in Schottland - eine 6-JahresStudie

Galea, G. (Edinburgh)

379 Bestimmung der Restantibiotika in kryogelagerten Herzklappen-Allotransplantaten

Jashari, R.; Faucon, F.; Van Hoeck, B.; De Gelas, S.; Fan, Y.; Vandenbulcke, S. (Brussels)

387 Der Einfluss von Hypothermie auf die Chondrozytenbeweglichkeit und KnorpelMatrixpermeabilität nach 1 Monat tiefgekühlter Lagerung

Brockbank, K.G.M. (North Charleston, SC/Atlanta, GA/ Charleston, SC); Rahn, E. (North Charleston, SC); Wright, G.J. (Charleston, SC); Chen, Z. (North Charleston, SC); Yao, H. (Charleston, SC)

\section{KARGER}

Fax +49 7614520714 


\section{Vol. 38, Issue 6, December 2011}

Letters to the Editors

393 Concerning von Auer F: To the Judgement of the European Court of Justice from December 9, 2010 concerning a National Rule That Bans the Import of Blood Products Which Do Not Originate from Blood Donations Completely Uncompensated. Transfus Med Hemother 2011;38:157

Lüder, S.R. (Hagen)

396 Reply to the Commentary of Dr. Sascha Rolf Lüder: Transfus Med Hemother 2011;38(6): DOI: 10.1159/000334844

Von Auer, F. (Königswinter)

350 Acknowledgement to Reviewers

397 Innovations

400 News / Ticker

402 Meetings and Conferences

403 Contents Vol. 38, 2011

386 Imprint

\section{Band 38, Heft 6, Dezember 2011}

Briefe an die Herausgeber

393 Zu von Auer F: Zum Urteil des Europäischen Gerichtshofes vom 9. Dezember 2010 zu einer nationalen Regelung, die die Einfuhr von Blutprodukten verbietet, die nicht aus gänzlich unbezahlt erfolgten Blutspenden stammen. Transfus Med Hemother 2011;38:157 Lüder, S.R. (Hagen)

396 Erwiderung zum Kommentar von Dr. Sascha Rolf Lüder: Transfus Med Hemother 2011;38(6): DOI: 10.1159/000334844

Von Auer, F. (Königswinter)

350 Dank an die Gutachter

397 Innovationen

400 News / Ticker

402 Tagungen und Kongresse

406 Inhalt Band 38, 2011-11-21

386 Impressum

\section{KARGER}

Fax +497614520714 